DOl $10.35381 / \mathrm{cm} . \mathrm{v} 5 \mathrm{i1} .321$

\title{
Incidencia en la aplicación de la NIC SP17 Propiedad, Planta y Equipo en las Empresas Municipales de Agua Potable
}

\section{Impact on the application of IAS SP17 Property, Plant and Equipment in Municipal Drinking Water Companies}

\author{
Dolores Viviana Ortiz Soto \\ dvortiz405@psg.ucacue.edu.ec \\ Universidad Católica de Cuenca, Cuenca \\ Ecuador \\ https://orcid.org/0000-0003-4752-0700 \\ Cecilia Ivonne Narváez Zurita \\ inarvaez@ucacue.edu.ec \\ Universidad Católica de Cuenca, Cuenca \\ https://orcid.org/0000-0002-7437-9880 \\ Juan Carlos Erazo Álvarez \\ jcerazo@ucacue.edu.ec \\ Universidad Católica de Cuenca, Cuenca \\ Ecuador \\ https://orcid.org/0000-0001-6480-2270
}

Recibido: 07 de septiembre de 2019

Aprobado: 28 de septiembre de 2019

\section{RESUMEN}

El artículo presenta un análisis de incidencias del tratamiento contable de propiedad, planta y equipo en la Empresa Municipal de Agua Potable El Tambo, a efectos de que los usuarios conozcan la relacionada con los cambios e inversiones realizados sobre estos rubros y tomen decisiones acertadas. La investigación se desarrolló bajo un enfoque mixto, en la primera etapa se realizó un análisis de la literatura especializada en el tema apoyada en herramientas cualitativas y en la segunda etapa se valoró la implementación de la NICSP 17 en la unidad de análisis a partir del uso de herramientas cuantitativas. Como resultado principal se estableció la deficiente determinación de la 
depreciación de los bienes y el no aprovechamiento de la deducibilidad de impuesto a la renta por este concepto.

Descriptores: Propiedad; Planta y equipo; Revalorización; Depreciación; Normas internacionales de información financiera, Toma de decisiones.

\begin{abstract}
The article presents an analysis of the incidences of the accounting treatment of property, plant and equipment in the Municipal Water Company El Tambo, in order that users of the financial statements can know the information related to the changes and investments made on these items and make wise decisions. The research was carried out under a mixed approach, in the first stage an analysis of the specialized literature on the subject supported by qualitative tools was carried out and in the second stage the implementation of IPSAS 17 in the unit of analysis from the use was assessed of quantitative tools. As a main result, the poor determination of the depreciation of the assets and the non-use of the income tax deductibility for this concept was established.
\end{abstract}

Descriptors: Property; Plant and equipment; Revaluation; Depreciation; International financial reporting standards; Decision making.

\title{
INTRODUCCIÓN
}

El proceso de armonización contable a nivel mundial llevado a cabo durante las últimas décadas ha facultado la consolidación de estándares internacionales, reflejados en las Normas Internacionales de Información Financiera (NIIF), su consolidación ha ido de la mano con una evolución y actualización vertiginosa, dando paso a una migración de la concepción de costo histórico a la concepción de valor razonable. Sin embargo, este escenario no ha progresado en las mismas condiciones en el ámbito tributario del Ecuador, ya que la normativa tributaria relacionada con el impuesto a la renta se mantiene aún anclada a la lógica del costo histórico.

Esta situación ha dado paso a la generación de diferencias entre la medición y el reconocimiento de ciertos elementos de los estados financieros tanto en las empresas públicas como en las privadas. Ante este contexto, el presente artículo tiene como 
objetivo principal realizar un análisis que muestre las dificultades de la medición y reconocimiento de propiedad, planta y equipo en la Empresa Municipal de Agua Potable El Tambo. El estudio se centra en las diferencias originadas entre las prácticas contables y la normativa tributaria relacionada con el impuesto a la renta en Ecuador.

\section{DESARROLLO}

\section{Normas internacionales de información financiera para el sector público.}

Partiendo de los precedentes establecidos por las Normas Internacionales de Contabilidad y el cambio de nombre de International Accounting Standards Committee ( IASC) a International Accounting Standards Board (IASB) las Normas Internacionales de Información Financiera (NIIF) surgen a raíz de la transformación en la estructura de emisión de estándares internacionales en el año 2001 como complemento a las Normas Internacionales de Contabilidad (NIC) y forman un conjunto de normas para la preparación de la información contable y presentación e interpretación de los estados financieros (Mondragón y Vásquez, 2013).

Las formalidades de las NIIF se ven reflejadas en la presentación de los estados financieros, en la eliminación de activos diferidos, depreciación integral de las revalorizaciones y ajustes de ejercicios anteriores, llevando a la aplicación cambios que garantizan seguridad en los registros y prestaciones de informes financieros. Chirinos (2016).

Estas normas legalmente exigibles, aceptadas a nivel mundial, comprensibles y de alta calidad están compuestas por un marco conceptual, que juega un papel importante en el desarrollo de los conceptos relacionados con la preparación y presentación de los estados financieros, y un conjunto de reglas y sus interpretaciones (Estupiñán, 2017).

Con lo antes indicado se debe acotar que las NIIF reflejan la esencia económica de las operaciones de una institución, en donde, su marco conceptual considera dos condicionales, la primera basado en el sistema de causación, la cual reconoce los 
eventos y transacciones, registrándolos en el momento que ocurren y son reportados en los estados financieros del ejercicio correspondiente, reflejándose las operaciones pasadas y aquellas que correspondan cancelar con recursos que se obtendrán a futuro o las que convengan hacer exigibles a favor de la institución y, la segunda considera que la empresa es creada para subsistir en el tiempo y no para liquidarla en el corto plazo (ibídem).

Las NIIF son reconocidas como un conjunto de normas internacionales para la preparación de los estados financieros, las cuales examinan y calculan los elementos que deben contener los activos, pasivos, patrimonio, ingresos y gastos (Rubiños 2015), información que al ser plasmada en un sistema contable compuesto por principios, interpretaciones y técnicas regula el comportamiento financiero de la institución (Borrero y Ortiz 2016). Con la aplicación de las NIIF los instrumentos financieros adquieren características como transparencia, oportunidad y relevancia, transformándose en una información financiera de calidad apta para la correcta toma de desiciones relacionandolas con estándares de contabilidad y auditoria a nivel mundial (Peña y Bastidas, 2007).

Con la implementación de las NIIF se aprecian factores benéficos tanto para sus agentes internos como externos, entre los cuales se encuentran:

- Incremento de la inversión internacional.

- Disponibilidad de un flujo de capitales con enfoque internacional.

- Garantía de la protección de los inversionistas procedentes de diversos sectores.

- Eliminación de procedimientos distintos para temas iguales.

- Transparencia y comparabilidad de la información financiera.

- Promoción de la normatividad internacional.

- Mejoramiento constantemente de la calidad de la información presentada en los estados financieros.

Las empresas necesitan el desarrollo de un modelo contable establecido 


\section{CIENCIAMATRIA}

Revista Interdisciplinaria de Humanidades, Educación, Ciencia y Tecnología

Año V. Vol. V. №1. Edición Especial. 2019

Hecho el depósito de ley: pp201602FA4721

ISSN-L: 2542-3029; ISSN: 2610-802X

Universidad Nacional Experimental Francisco de Miranda (UNEFM). Santa Ana de Coro. Venezuela

Dolores Viviana Ortiz Soto; Cecilia Ivonne Narváez Zurita: Juan Carlos Erazo Álvarez

internacionalmente que refleje la realidad financiera de las mismas; un modelo que les encamine a generar competitividad, adquirir sistemas de información transparentes y de buena calidad, permitiéndoles una acertada toma de decisiones tanto a nivel operativo como financiero (Solís, 2012) y para ello se requiere encaminar el ajuste de los sistemas de información internos a cada una de las NIIF vigentes (Tabla 1)

\section{Tabla 1.}

NIIF vigentes 
CIENCIAMATRIA

Revista Interdisciplinaria de Humanidades, Educación, Ciencia y Tecnología

Año V. Vol. V. №1. Edición Especial. 2019

Hecho el depósito de ley: pp201602FA4721

ISSN-L: 2542-3029; ISSN: 2610-802X

Universidad Nacional Experimental Francisco de Miranda (UNEFM). Santa Ana de Coro. Venezuela

Dolores Viviana Ortiz Soto; Cecilia Ivonne Narváez Zurita: Juan Carlos Erazo Álvarez

\begin{tabular}{|c|c|}
\hline \multicolumn{2}{|r|}{ Normas Vigentes } \\
\hline \multicolumn{2}{|r|}{ NIIF - Norm as Internacionales de Inform ación Financiera } \\
\hline NIIF 1 & $\begin{array}{l}\text { Adopción por primera vez de las Normas Internacionales de } \\
\text { Información Financiera }\end{array}$ \\
\hline NIIF 2 & Pagos basados en acciones \\
\hline NIIF 3 & Combinaciones de negocios \\
\hline NIIF 4 & Contratos de seguro \\
\hline NIIF 5 & $\begin{array}{l}\text { Activos no corrientes mantenidos para la venta y operaciones } \\
\text { discontinuadas }\end{array}$ \\
\hline NIIF 6 & Exploración y evaluación de recursos minerales \\
\hline NIIF 7 & Instrumentos financieros; Información a revelar \\
\hline NIIF 8 & Segmentos de operación \\
\hline NIIF 9 & Instrumentos financieros \\
\hline NIIF 10 & Estados financieros consolidados \\
\hline NIIF 11 & Acuerdos conjuntos \\
\hline NIIF 12 & Información a revelar sobre participaciones en otras entidades \\
\hline NIIF 13 & Medición del valor razonable \\
\hline NIIF 14 & Cuentas de diferimientos de actividades reguladas \\
\hline NIIF 15 & $\begin{array}{l}\text { Ingresos de actividades ordinarias procedentes de contratos con } \\
\text { clientes }\end{array}$ \\
\hline NIIF 16 & Arrendamientos \\
\hline
\end{tabular}

NIC - Norm as Internacionales de Contabilidad

NIC 1 Presentación de estados financieros

NIC 2 Inventarios

NIC 7 Estado de flujos de efectivo

NIC 8 Políticas contables, cambios en las estimaciones contables y errores

\section{Normas Internacionales de Contabilidad del Sector Público, NICSP}

"Las Normas Internacionales de Contabilidad para el Sector Público (NICSP) han tenido un gran impacto a nivel mundial, se han ido enfocando en la Contabilidad Gubernamental en el sector público dándole un cambio a los estados financieros, teniendo una 
información confiable, oportuna para la toma de decisiones en las instituciones $u$ organizaciones" (Morán y Román, 2018, pág. 3)

Las NICSP se basan considerablemente en las NIIF, y son las únicas normas reconocidas a nivel mundial con aplicación en el sector público, de tal manera que las NIIF son utilizadas por las empresas públicas y las NICSP por el resto de instituciones del sector público (Pimienta y Pessoa, 2015). Por otro lado, las NICSP no se enfocan únicamente al tema contable, sino también al patrimonio público examinando los valores en las entidades gubernamentales; durante los últimos años, la aplicación de las NICSP ha proporcionado un proceso firme y acumulativo; convirtiéndose en un elemento propicio en lo referente a la toma de decisiones y el cambio de caja al devengo, Arraiz (2018).

En este contexto, se resalta la NIC 16, propiedades, planta y equipo, por ser considera la NIIF objeto de investigación de este artículo. Dicha norma tiene por finalidad constituir el proceso contable de propiedades, planta y equipo, de tal manera que los usuarios de los estados financieros tengan conocimiento de la información relacionada con la inversión que la empresa ha realizado en sus propiedades, planta y equipo, así como los cambios que se hayan generado en esta inversión. Para ello, se entenderá a este grupo de cuentas contables como los activos tangibles de propiedad de una empresa que pueden ser utilizados en la producción o para ser arrendados a terceros o con fines administrativos, y aquellos que se utilizaran por más un ejercicio económico.

La NIC 16, determina los procedimientos contables de propiedad planta y equipo, de tal manera que se conozca la inversión y los cambios que la entidad dispone en esta cuenta, reflejándolos en los estados financieros, así mismo, permite realizar el reconocimiento, contabilización de los activos, determinar su importe en libros y los cargos por depreciación y pérdidas por deterioro.

Considerando que el activo forma parte de la estructura de algunos instrumentos financieros Macedo ( 2007), Accid (2005), Fierro y Fierro (2015) clasifican al activo por su grado de liquidez, proporcionando las características propias de su categorización, 
razón que le da a los activos corrientes el primer lugar por disponer de un gran número de operaciones con la facilidad de convertirse inmediatamente en efectivo, seguido se encuentra los activos fijos conformado por propiedad, planta y equipos que fueron adquiridos por la empresa con la finalidad de generar producción, propiciando estabilidad a la misma, finalmente los activos diferidos de los que se pretende recibir bienes 0 servicios para ser consumidos en el futuro.

Siendo Propiedad, Planta y Equipo el segundo grupo del activo, regulado bajo la NIC 16, se considera diversos componentes para el registro contable de sus elementos:

- Reconocimiento

Para identificar a un elemento de propiedad planta y equipo, se requiere poseer sensatez para establecer criterios y clasificarlos, a fin de que en un futuro se obtengan beneficios económicos de estos.

Las características adicionales con las que debe contar un activo fijo se las determina en el costo del mismo, el cual se mide con eficacia al inicio de la adquisición, considerando todos los aranceles por la importación e impuestos indirectos que no se pueda recuperar además de deducir todas las rebajas del importe a pagar; por otra parte se debe estimar los desembolsos monetarios que se efectúe para ubicar el bien en un lugar adecuado para su operatividad, así como también, costos que se incurre al final para cambiar o conservar el elemento correspondiente del activo.

- Medición posterior al reconocimiento

Una vez clasificado un activo, la entidad está en libertad de elegir el modelo de medición más conveniente de acuerdo a sus características y aplicarlo como parte de sus políticas contables. El valor razonable del activo fijo deberá ser registrado a su valor revaluado, el mismo que, corresponde al valor razonable al momento de la revaluación menos la depreciación acumulada dando lugar a la utilización del modelo de revaluación. Por otro lado, si el activo fijo se contabiliza al costo histórico menos la depreciación acumulada y el total de las pérdidas generadas por deterioro se está utilizando el modelo del costo. 


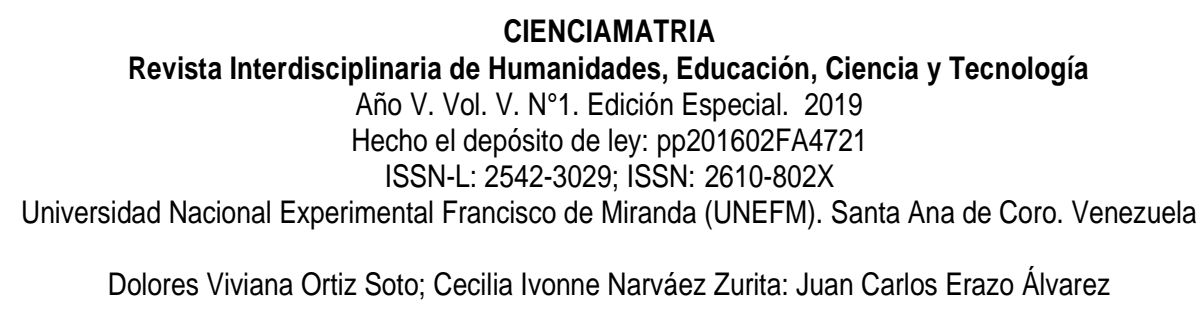

Con el propósito de regular el importe en libros y el valor razonable es necesario realizar una evaluación periódica de la cual se obtendrá un aumento o disminución del importe en libros, en el primer caso del aumento se debe identificar en el resultado integral y se reflejará en el patrimonio como superávit de revaluación del activo, en cambio en la disminución se reconocerá en otro resultado integral (Accid, 2005).

- Depreciación

El método de depreciación aplicado por la empresa debe ser revisado por lo menos al término de cada periodo anual y en él se reflejará el patrón por el cual la empresa ha optado sean consumido los beneficios económicos futuros del activo, en el caso de que se registre un cambio en el patrón este debe ser contabilizado como un cambio en la estimación contable según señala la NIC 8 (International Financial Reporting Standards -IFRS).

La depreciación de un activo se lo realiza en el momento en el que se encuentre en el lugar y en las condiciones favorables para su operatividad, y finalizará cuando se proceda con la baja de las cuentas y se lo haya catalogado para venta o donación, en tal virtud, se pueden mencionar tres métodos de depreciación: Método lineal, creciente y por unidades producidas, sin embargo, la empresa analizará y empleará el método considerando la forma en la que se irá consumiendo los beneficios económicos generados por el activo, debiendo también, estar consciente de que como empresa pública en el Ecuador a través del Órgano Generador y Recaudador de los Tributos (SRI), emite consideraciones para la estimación de porcentajes de depreciación estipulados en el Art. 28 del Reglamento de Régimen Tributario Interno detallados (Dirección Nacional Jurídica , 2015) a continuación: 
Dolores Viviana Ortiz Soto; Cecilia Ivonne Narváez Zurita: Juan Carlos Erazo Álvarez

I. Inmuebles (excepto terrenos), naves, aeronaves, barcazas y similares $5 \%$ anual

II. Instalaciones, maquinarias, equipos y muebles $10 \%$ anual

III. Vehículos equipos de transporte, equipo caminero móvil $20 \%$ anual

IV. Equipos de computación y software $33 \%$ anual.

- Baja de cuentas

Cuando se deja de percibir beneficios económicos futuros de un activo o estos a su vez están listos para enajenación la pérdida o ganancia generada por este concepto se lo realiza en el primer caso con inclusión en el resultado del ejercicio siempre y cuando la partida se haya dado de baja en cuentas, y, cuando se registre una ganancia esta no se considerará como parte de los ingresos ordinarios; además de que la diferencia obtenida por la baja de cuentas se determinará entre el importe neto obtenido por la enajenación y el importe en libros del elemento considerado como punto referencial para determinar la pérdida o ganancia de la baja en cuentas.

Con la finalidad de lograr un buen manejo de los activos fijos, Córdoba (2016) considera que implementar la gestión integral que involucra a los departamentos de finanzas y mantenimiento a través de la sincronización de sus bases de datos permite al área contable implementar las NIIF para el adecuado control de los activos fijos, a efectos de conseguir una gestión eficiente y valuar su impacto en la situación financiera y de resultados de la empresa.

- Deterioro del valor de los activos

La NIC 36 asegura la contabilización de los activos cuando se presenta una pérdida por deterioro, esta pérdida se da cuando su importe en libros excede a su importe recuperable, estos indicios pueden verificarse por dos tipos de fuentes internas y externas de información. 


\author{
CIENCIAMATRIA \\ Revista Interdisciplinaria de Humanidades, Educación, Ciencia y Tecnología \\ Año V. Vol. V. №1. Edición Especial. 2019 \\ Hecho el depósito de ley: pp201602FA4721 \\ ISSN-L: 2542-3029; ISSN: 2610-802X \\ Universidad Nacional Experimental Francisco de Miranda (UNEFM). Santa Ana de Coro. Venezuela \\ Dolores Viviana Ortiz Soto; Cecilia Ivonne Narváez Zurita: Juan Carlos Erazo Álvarez
}

\title{
Fuentes externas
}

I. Indicios observables de la disminución del valor de los activos durante un periodo a parte de los presentados por su vida útil.

II. Durante un periodo las tasas de interés del mercado y por rendimientos financieros, afecten la tasa de descuento para calcular el valor del uso del activo de tal manera que disminuye significativamente su importe.

III. Cuando el valor en libros de los activos de la empresa sea superior al de su capitalización bursátil.

\section{Fuentes internas}

I. Deterioro físico u obsoleto de un activo.

II. Variación en la forma en que se espera hacer uso del activo afectando negativamente a la empresa, estos cambios incluyen lo siguiente: la inoperatividad del bien; discontinuación o restructuración de la operatividad del activo; planes para usar el activo antes de la fecha estipulada; $y$, reconsideración como finita la vida útil de un activo.

Además, la NIC 36 señala que se debe comprobar por lo menos una vez al año el desgaste del valor de los activos intangibles con una vida útil indefinida, así como de los activos que no se encuentran habilitados para su uso, comparando su costo en libros con su costo recuperable.

- Información a revelar

Se deberá revelar información en los estados financieros relacionada a cada una de las clases de propiedades, planta y equipo, de acuerdo a los siguientes aspectos:

I. Bases de medición utilizadas en la determinación del importe en libros.

II. Métodos de depreciación aplicados.

III. Vida útil y/o tasas de depreciación. 
IV. Importe bruto en libros y depreciación acumulada.

V. Conciliación entre los valores iniciales en libros y los valores al final del periodo.

VI. Importes con restricciones de titularidad.

\section{METODOLOGÍA}

La investigación tuvo un enfoque mixto y un alcance explicativo secuencial, para ello, se empleó en la primera etapa herramientas de carácter cualitativo en la descripción de las características de la contabilidad gubernamental y de la NIC 16 Propiedades, planta y equipo, y en la segunda etapa factores cuantitativos puesto que a través del procesamiento de datos con el uso de la estadística inferencial se determinó la problemática que atraviesa la unidad de análisis. La metodología que se utilizó fue el estudio de caso aplicado a la Empresa Municipal de Agua Potable y Alcantarillado El Tambo de la provincia de Cañar, Ecuador. La actividad principal de esta empresa pública es el suministro del servicio de agua potable y el mantenimiento y operación del sistema de alcantarillado. La finalidad de la investigación fue de tipo transversal ya que los datos fueron recopilados en un único momento del tiempo. Se aplicaron como técnicas de investigación: la entrevista, que fue dirigida al Gerente de la EMAPAT-EP con el propósito de conocer su criterio sobre ciertos aspectos contables de propiedad, planta y equipo; la encuesta, se dirigió al Consejo Directivo, gerente y personal del departamento contable de la empresa, con base en un cuestionario estructurado que permitió recabar información relacionada con las políticas contables, adquisición y baja de activos fijos, depreciación, vida útil , obsolescencia, mantenimiento, revalorización, valor residual, revelación de información en los estados financieros y gastos deducibles.

\section{RESULTADOS}

1. A partir de la aplicación de los instrumentos de investigación se presentan los siguientes resultados: 
$\checkmark$ Inversión en propiedad planta y equipo: la planificación de inversiones es un componente central para llevar a cabo el plan estratégico de toda organización, en este sentido, se determinó que la EMAPAT-EP no cuenta con un plan de inversión en propiedades, planta y equipo, por lo que las adquisiciones se realizan de acuerdo a las necesidades que surgen en cada departamento, inobservando el impacto que genera estas acciones sobre sus costos.

$\checkmark$ Constatación física de propiedades, planta y equipo: este procedimiento consiste en verificar el estado en el que se encuentran los bienes que posee la entidad, al respecto, se verificó que la empresa no cuenta con procedimientos documentados para la constatación física, por lo que, la constatación se realiza de una manera poco técnica y sin considerar los cierres de los ejercicios contables.

$\checkmark$ Actividades de control: las actividades de control permiten evaluar la situación actual respecto a la gestión de propiedades, planta y equipo, bajo esta consideración, se estableció que los activos de la empresa carecen de un etiquetado y de la identificación de su ubicación física.

$\checkmark$ Procedimientos de adquisición: los procedimientos inherentes a la adquisición de activos fijos deben asegurar que su desarrollo se realice en cumplimiento de las metas y objetivos planteados por la entidad, en este sentido, se determinó que las compras de bienes se realizan bajo la ley de contratación pública con el respectivo respaldo de su comprobante de venta legal y su registro contable oportuno.

$\checkmark$ Vida útil: la vida útil corresponde al tiempo de operatividad de un activo determinado por la empresa, en cuanto a este parámetro, existe falta de control sobre la vida útil y depreciación de los activos fijos, ya que la empresa no está cumpliendo con la técnica contable adoptada, en este sentido, hay bienes como un vehículo adquirido en el año 2008, mobiliarios y equipo de cómputo adquiridos 


\section{CIENCIAMATRIA}

Revista Interdisciplinaria de Humanidades, Educación, Ciencia y Tecnología

Año V. Vol. V. N¹. Edición Especial. 2019

Hecho el depósito de ley: pp201602FA4721

ISSN-L: 2542-3029; ISSN: 2610-802X

Universidad Nacional Experimental Francisco de Miranda (UNEFM). Santa Ana de Coro. Venezuela

Dolores Viviana Ortiz Soto; Cecilia Ivonne Narváez Zurita: Juan Carlos Erazo Álvarez

en el año 2012, que terminaron su vida útil y que en los estados financieros del año 2018 reportan aún un rubro por la depreciación anual.

$\checkmark$ Depreciación: corresponde al mecanismo a través del cual es posible reconocer el desgaste de los bienes contable y financieramente. La depreciación de los activos tiene un impacto financiero y tributario en las empresas, ya que el gasto de depreciación debe ser llevado al estado de resultados, en consecuencia, la utilidad se ve disminuida al igual que el pago del impuesto a la renta en el caso del Ecuador, y finalmente, tiene un efecto directo sobre la estructura financiera al disminuir el valor de los bienes, bajo este contexto, se determinó que la empresa no está realizando correctamente las depreciaciones (ver tabla 2), ya que ha optado por el método de línea recta, sin embargo, la depreciación registrada en libros correspondiente a vehículos y equipos y paquetes informáticos solo refleja el valor residual más no el valor de la depreciación, por consiguiente, en los estados financieros no está reflejando el valor real de los elementos de propiedades, planta y equipo, mientras que, en el ámbito tributario no se está aprovechando la deducibilidad integral por el gasto de depreciación, tal como dispone la Ley de Régimen Tributario Interno.

\section{Tabla 2. \\ Depreciación acumulada de propiedades, planta y equipo}


CIENCIAMATRIA

Revista Interdisciplinaria de Humanidades, Educación, Ciencia y Tecnología

Año V. Vol. V. N¹. Edición Especial. 2019

Hecho el depósito de ley: pp201602FA4721

ISSN-L: 2542-3029; ISSN: 2610-802X

Universidad Nacional Experimental Francisco de Miranda (UNEFM). Santa Ana de Coro. Venezuela

Dolores Viviana Ortiz Soto; Cecilia Ivonne Narváez Zurita: Juan Carlos Erazo Álvarez

\begin{tabular}{lccc}
\hline Propiedad, & Valor & Valor & Gasto No \\
Planta y Equipo & $\begin{array}{c}\text { Depreciación } \\
\text { EMAPAT-EP }\end{array}$ & $\begin{array}{c}\text { Depreciación } \\
\text { Deducible }\end{array}$ &
\end{tabular}

Según NIC

\begin{tabular}{llll}
\hline $\begin{array}{l}\text { Maquinaria y } \\
\text { Equipo }\end{array}$ & $\$ 2.837,00$ & $\$ 2.837,00$ & $\$-$ \\
\hline Vehículo & $\$ 1.509,91$ & $\$ 2684,29$ & $\$-1174,38$ \\
& & & \\
\hline $\begin{array}{l}\text { Equipos y } \\
\text { Paquetes }\end{array}$ & $\$ 1.257,12$ & $\$ 1559,76$ & $\$-302,64$
\end{tabular}

2. Análisis de las diferencias originadas a partir de las normas contables y de las normas tributarias vigentes en los elementos de propiedad, planta y equipo

\begin{tabular}{|l|l|}
\hline $\begin{array}{l}\text { Tratamiento dispuesto en las Normas } \\
\text { Tributarias (LORTI) }\end{array}$ & $\begin{array}{l}\text { Tratamiento contable establecido en } \\
\text { las NIIF }\end{array}$ \\
\hline \multicolumn{2}{|c|}{ Costo } \\
\hline $\begin{array}{l}\text { Se refiere al importe en efectivo, otros } \\
\text { medios líquidos o el valor razonable de la } \\
\text { contraprestación entregada para adquirir } \\
\text { un activo o para su construcción por parte } \\
\text { de la empresa. En el caso de que el activo } \\
\text { sea construido por la empresa, su costo } \\
\text { puede estar dado por las transacciones por } \\
\text { terceros para comprar materiales, mano de } \\
\text { obra y otros factores necesarios para la } \\
\text { construcción. }\end{array}$ & \\
\hline $\begin{array}{l}\text { El importe en libros se refiere al valor que } \\
\text { es reconocido el bien una vez que se haya } \\
\text { procedido a deducir la depreciación } \\
\text { acumulada y las pérdidas por deterioro en } \\
\text { caso de presentarse. }\end{array}$ & \\
\hline
\end{tabular}




\section{Depreciación}

Corresponde a la distribución sistémica del importe depreciable de un activo durante su vida útil.

\section{Deducción de la depreciación}

El valor de depreciación de cada periodo se reconocerá como gasto en dicho periodo, no obstante, los beneficios económicos incorporados a que la empresa utilice para la producción de otros activos, no da origen a un gasto en el periodo.

La depreciación de los activos fijos se debe ejecutar en concordancia con su naturaleza, con la duración de su vida útil y la técnica contable adoptada. Por consiguiente, el gasto será deducible siempre que no supere los siguientes porcentajes: -Inmuebles 5\% anual.

-Instalaciones, maquinarias, equipos y muebles $10 \%$ anual.

-Vehículos, equipos de transporte y equipo caminero móvil $20 \%$ anual. -Equipos de cómputo y software 33\% anual.

\section{Valor razonable}

\section{El valor razonable representa el importe No aplica}

por el cual puede ser intercambiado un activo entre partes interesadas, quienes disponen de la información necesaria para realizar una transacción libre.

\section{Pérdida por deterioro}

Se considera deterioro de un activo cuando el importe en los registros contables es superior a su importe recuperable, en tal razón, el gasto por deterioro se reconocerá cuando su valor en libros no pueda ser recuperado totalmente por la entidad.
En caso de deterioro acelerado, el respectivo Director Regional del Servicio de Rentas Internas, será la persona encargada de autorizar depreciaciones en porcentajes superiores a los estipulados en la LORTI, considerando la técnica contable y los parámetros técnicos de cada industria y del respectivo bien.

\section{Valor residual de un activo}




\begin{abstract}
Corresponde al importe neto estimado que una organización espera obtener de un elemento de propiedad, planta y equipo una vez que ha concluido su vida útil y se han deducido aquellos costos derivados de la desapropiación.
\end{abstract}

La LORTI no reconoce el valor residual de los activos, puesto que financieramente el valor residual no se deprecia. Este elemento se identifica sólo para el cálculo del IVA en caso de arrendamientos mercantiles.

\section{Vida útil}

La vida útil corresponde al período en el cual se espera que la entidad haga uso del activo, la vida útil puede ser identificada además en función del número de unidades de producción que se espera obtener del bien.

\section{Revaluaciones}

\section{El incremento del importe en libros de los} activos por un proceso de revaluación, se reconocerá en otro resultado integral y se registrará en el patrimonio como superávit de revaluación, mientras que, la reducción del importe en libros por la revaluación se reconocerá en el resultado del período.
La LORTI no presenta estimaciones puntuales de la vida útil, sin embargo, identifica los porcentajes de depreciación.

proceda a revaluar sus activos, la
depreciación correspondiente a
dicho revalúo no será considerada
como gasto deducible. En el caso de
asignarse un nuevo valor a los
activos completamente depreciados,
no deberán volver a depreciarse.
Si se produjere la venta de bienes
revaluados se asumirá como ingreso


gravable la diferencia entre el precio de venta y el valor residual sin considerar el revalúo.

\section{CONCLUSIONES}

$\checkmark$ La NIC 16 establece el tratamiento para el registro contable de propiedad, planta y equipo, dicho proceso posibilita a los usuarios internos y externos de los estados financieros de una empresa conocer las inversiones que se han realizado, así como los cambios que se han efectuado por deterioro, obsolescencia, mantenimiento, mejoras y bajas.

$\checkmark$ Entre los principales problemas evidenciados en EMAPAT-EP con relación al procedimiento contable de propiedad, planta y equipo son: la determinación del importe en libros y los cargos por depreciación, ya que no se consideró adecuadamente los años de vida útil de los activos en base a su naturaleza.

$\checkmark \quad$ A fin de evitar una disrupción entre la Ley de Régimen Tributario Interno y la NIC 16 es recomendable que las empresas establezcan una fecha de legalización de sus activos fijos, ya que esta determina el cumplimiento del registro contable de la depreciación del activo fijo dentro de un ejercicio contable, en consecuencia, este procedimiento faculta a que la depreciación sea considerada como un gasto deducible al cumplir con el requisito previsto en el artículo 22 del Reglamento de la LORTI

$\checkmark$ En base a los resultados expuestos en la investigación, la falta de una planificación de adquisición de activos fijos y políticas de control de estos, perjudican el control constante de propiedad, planta y equipo, ocasionando que la información financiera de la empresa no resulte fiable, obstaculizando el desarrollo de la misma.

$\checkmark$ De igual manera, en base a los resultados, el no mantener una constante actualización en cuanto al control de los años de vida útil y porcentajes de 
depreciación de los bienes establecidos por la LORTI, genera inconsistencias en el registro de las depreciaciones arrojando resultados equívocos en cuanto a costos y gastos que la empresa debe incurrir.

$\checkmark \quad$ Finalmente, queda expuesto el problema entre la armonización de las NIIF y la legislación tributaria ecuatoriana, ya que el cambio radical de llevar la contabilidad para efectos directamente fiscales, y que ahora el objetivo sea mostrar la realidad económica de la empresa que se aleja de los intereses impositivos, genera un fuerte conflicto con la administración tributaria del país, principalmente en aspectos relacionados con las depreciaciones, vida útil y costos de desmantelamiento, ya que estos impactarán claramente sobre las ventajas que tenía el Servicio de Rentas Internas tanto para recaudar como para controlar.

\section{REFERENCIAS CONSULTADAS}

1. Accid, P., (2005). Normas internacionales de contabilidad NIC NIIF. Grupo Planeta (GBS).

2. Arraiz, A. (2018). Framerwork de desarrollo de Proyectos Sociotecnológicos basado en la notación de Metamodelos de procesos de Ingeniería de Software (spem 2.0). Revista Arbitrada Interdisciplinaria Koinonía, 3(6), 79-95. Recuperado de

https://fundacionkoinonia.com.ve/ojs/index.php/revistakoinonia/article/view/141/1 $\underline{21}$

3. Borrero, A., \& Ortiz, O. (2016). PYMES: implementación de normas de información financiera internacional. (2. Universidad del Norte, Ed.) Barranquilla, Colombia.

4. Córdoba, M. (2016). Gestión Financiera. Bogotá: EcoEdiciones. 


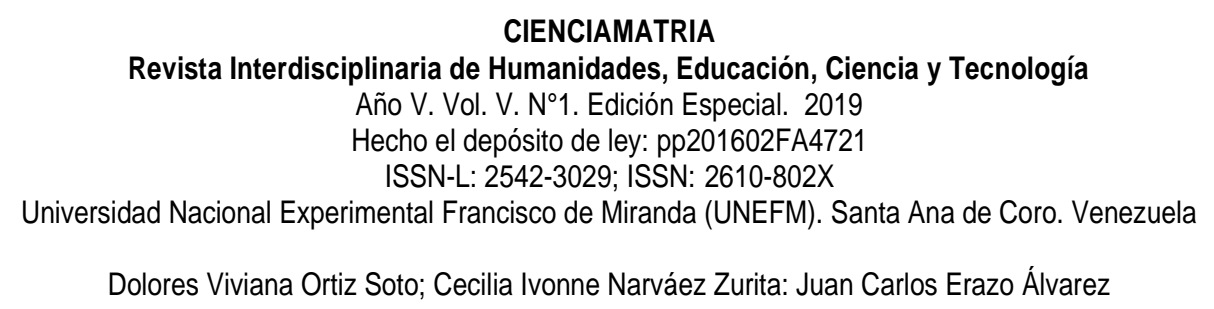

5. Chirinos, A. (2016). La innovación como factor clave de éxito en la gerencia de las empresas de producción social venezolana. CIENCIAMATRIA, 2(2), 97-106. https://doi.org/10.35381/cm.v2i2.55

6. Dirección Nacional Jurídica. (2015). Reglamento para la aplicación de la Ley de régimen Tributario Interno. Quito: Departamento de Normativa.

7. Estupiñán, R. (2017). Estados Financieros Bajo Las NIC- NIIF. Colombia: EcoEdiciones.

8. Fierro, A., \& Fierro, F. (2015). Contabilidad de Activos con efoque NIIF para pymes (Vol. 3). Bogotá: Ecoe Ediciones.

9. Macedo, J. j. (2007). Introducción a la Contabilidad. Zapopan, Jalisco, Mexico.

10. Mondragón, M., \& Vásquez, N. (2013). Normas Internacionales de Información Financiera. Barcelona: Profit Editorial

11. Morán Ortega, M. F., \& Román Tomalá, J. M. (2018). Análisis de Normas Internacionales de Contabilidad para el Sector Público en Ecuador. Milagro.

12. Peña, A., \& Bastidas, M. (2007). La ética: fundamento en la adopción de las normas internacionales de contabilidad. Actualidad Contable Faces vol 10.

13. Pimienta, C., \& Pessoa, M. (2015). Gestión financiera pública en América Latina. Fast lines Inc.

14. Rubiños, W. $(2015,11)$. Aportes y Ventajas de las NIIF. Building a better working world.

15. Solís, V. (2012). Cumplimiento de las disposiciones legales y su efecto en la presentación de la información financiera con aplicación de la NIIF para Pymes en Retrounic del Ecuador S.A. en el periodo de transición 2011 y aplicación 2012. Visión General.

\section{REFERENCES CONSULTED}

1. Accid, P., (2005). International accounting standards IAS IFRS. Grupo Planeta (GBS). 


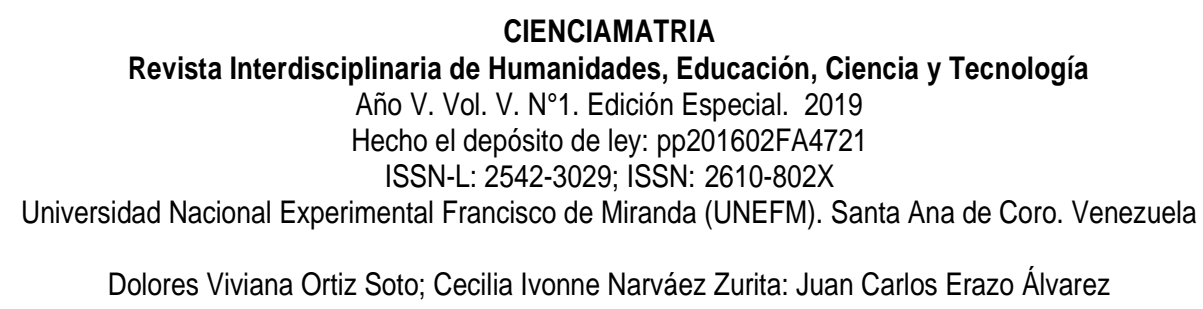

2. Arraiz, A. (2018). Framerwork of development of Sociotechnological Projects based on the notation of Metamodels of Software Engineering processes (spem 2.0). Interdisciplinary Arbitrated Review Koinonía, 3 (6), 79-95. Recovered from https://fundacionkoinonia.com.ve/ojs/index.php/revistakoinonia/article/view/141/1 $\underline{21}$

3. Borrero, A., \& Ortiz, O. (2016). SMEs: implementation of international financial information standards. (2. Universidad del Norte, Ed.) Barranquilla, Colombia.

4. Córdoba, M. (2016). Financial management. Bogotá: EcoEdiciones.

5. Chirinos, A. (2016). Innovation as a key success factor in the management of Venezuelan social production companies. SCIENCE, 2 (2), 97-106. https://doi.org/10.35381/cm.v2i2.55

6. National Legal Department. (2015). Regulations for the application of the Internal Tax Regime Law. Quito: Regulatory Department.

7. Estupiñán, R. (2017). Financial Statements Under IAS-IFRS. Colombia: EcoEdiciones.

8. Fierro, A., \& Fierro, F. (2015). Asset Accounting with efoque IFRS for SMEs (Vol. 3). Bogotá: Ecoe Editions.

9. Macedo, J. j. (2007). Introduction to Accounting. Zapopan, Jalisco, Mexico.

10. Mondragón, M., \& Vásquez, N. (2013). International Financial Reporting Standards. Barcelona: Profit Editorial

11. Morán Ortega, M. F., \& Román Tomalá, J. M. (2018). Analysis of International Accounting Standards for the Public Sector in Ecuador. Miracle.

12. Peña, A., \& Bastidas, M. (2007). Ethics: foundation in the adoption of international accounting standards. Accounting Faces vol 10.

13. Pimienta, C., \& Pessoa, M. (2015). Public financial management in Latin America. Fast lines Inc.

14. Rubiños, W. $(2015,11)$. Contributions and Advantages of IFRS. Building a better working world. 


\section{CIENCIAMATRIA}

Revista Interdisciplinaria de Humanidades, Educación, Ciencia y Tecnología

Año V. Vol. V. №1. Edición Especial. 2019

Hecho el depósito de ley: pp201602FA4721

ISSN-L: 2542-3029; ISSN: 2610-802X

Universidad Nacional Experimental Francisco de Miranda (UNEFM). Santa Ana de Coro. Venezuela

Dolores Viviana Ortiz Soto; Cecilia Ivonne Narváez Zurita: Juan Carlos Erazo Álvarez

15. Solís, V. (2012). Compliance with legal provisions and their effect on the presentation of financial information with the application of the IFRS for SMEs in Retrounic del Ecuador S.A. in the 2011 transition period and 2012 application. Overview.

(C2019 por los autores. Este artículo es de acceso abierto y distribuido según los términos y condiciones de la licencia Creative Commons Atribución-NoComercial-Compartirlgual 4.0 Internacional (CC BY-NC-

SA 4.0) (https://creativecommons.org/licenses/by-nc-sa/4.0/). 